\title{
A Study Comparing Different Chemoradiation Techniques in the Management of Locally Advanced Head and Neck Squamous Cell Cancers
}

\author{
Suman Ghorai ${ }^{1}$, Bikramjit Chakrabarti ${ }^{2}$ \\ ${ }^{1}$ Department of Radiotherapy, Bankura Sammilani Medical College, West Bengal, India. \\ ${ }^{2}$ Department of Radiotherapy, Institute of Post-Graduate Medical Education and \\ Research, Kolkata, West Bengal, India.
}

\section{ABSTRACT}

\section{BACKGROUND}

Concomitant chemoradiation is the treatment of choice for most of head and neck cancer patients in developing countries since these patients present in locally advanced stage. They account for $4 \%$ of all cancers. Despite treatment, loco-regional failure is seen in $51-59 \%$ patients. There is often a deferral in start of radiation therapy due to large number of cases and lesser number of existing radiation set ups. Hence, several oncologists use neo-adjuvant systemic therapy prior to external beam radiotherapy. Some oncologists prefer hypofractionated radiation therapy over conventional fractionation. Yet there is no data available comparing neoadjuvant chemotherapy followed by hypofractionation with usual concurrent chemoradiation.

\section{METHODS}

Biopsy proved squamous cell carcinoma of larynx and pharynx of stage III \& IV excluding IVC were incorporated in this study. Patients attending Bankura Sammilani Medical College Hospital in West Bengal, were randomized to get either concurrent chemoradiation with concomitant weekly injection of cisplatin $40 \mathrm{mg} / \mathrm{m}^{2}$ along with an external beam radiotherapy (EBRT) dose of $70 \mathrm{~Gy}$. in conventional dose fractionation; or neoadjuvant chemotherapy for three cycles with carboplatin AUC 6, injection paclitaxel $175 \mathrm{mg} / \mathrm{m}^{2}$ and injection 5-fluorouracil $1000 \mathrm{mg} / \mathrm{m}^{2}$ day 1-4 followed by hypo-fractionated EBRT ( 40 Gy. in 16 fractions for 3.5 weeks followed by boost dose of 20 Gy. over 8 fractions in 1.5 weeks) commenced within 3 weeks after completion of chemotherapy. Disease-free survival (DFS) at three years and complications of treatment (using version 5.0 Common-Terminology-Criteria for Adverse-Events) were compared statistically using one-way ANOVA by means of version 20 IBM SPSS Statistics ${ }^{\circledR}$ software.

\section{RESULTS}

Characteristics of patients were comparable in the two arms. Acute toxicities were less after neoadjuvant chemotherapy followed by hypo fractionated radiotherapy arm. DFS and tumor control were same. Lesser treatment days made this arm more convenient to patients. This is also financially helpful in Bankura where poor patients have to stay near hospital in some rented room or they have to come regularly from long distance by hired vehicle or ambulance. Tumor control and survival advantage was same in both modalities of treatment. Thus hypo fractionated radiotherapy arm was non-inferior statistically when compared to concomitant arm in terms of tumor control and survival.

\section{CONCLUSIONS}

Management by neo-adjuvant chemotherapy followed by hypo-fractionated radiation is a non-inferior apposite substitute to the usual concurrent chemoradiation in cancer treatment centers overburdened with patients.

\section{KEY WORDS}

Head and Neck, Cancer, Radiation, Fractionation
Corresponding Author: Bikramjit Chakrabarti, Room No. 2, Department of Radiotherapy, IPGMER and SSKM Hospital, $244<$ AJC Bose Road, Kolkata-700020, West Bengal, India.

E-mail:jtbkm@yahoo.com

DOI: $10.14260 /$ jemds/2020/114

Financial or Other Competing Interests: None.

How to Cite This Article:

Ghorai S, Chakrabarti B. A study comparing different chemoradiation techniques in the management of locally advanced head and neck squamous cell cancers. J. Evolution Med. Dent. Sci. 2020;9(08):508-511, DOI: 10.14260/jemds/2020/114

Submission 07-11-2019,

Peer Review 28-01-2020,

Acceptance 05-02-2020,

Published 24-02-2020. 


\section{BACKGROUND}

Head-neck cancers are one of the most common cancers worldwide. They account for $4 \%$ of all cancers. It is predicted that over 65,000 patients will be identified in United States in 2017.[1] Despite treatment, loco-regional failure is seen in 5159\% patients. ${ }^{[2]}$ Around $27 \%$ of them are women. ${ }^{[3]} \mathrm{A}$ higher age-adjusted incidence is seen in Afro-Americans than other races. Contemporary multimodal treatment of head and neck cancer can involve surgery and/or intensity modulated radiation therapy (IMRT) with or without platinum-based chemotherapy. Surgery often involves resection of the primary tumour as well as neck dissection historically classified as radical or selective (preserves one or more lymph node level normally removed in a radical neck dissection). In general, the more extensive neck dissections result in more severe shoulder dysfunction, pain, and reduced quality of life. Although resection or damage to the spinal accessory nerve is a major cause, this morbidity, damage to other key structures, including the cervical root branches and cervical plexus, also plays an important role. Perturbation of scapulothoracic motion from trapezius weakness (compromised SAN) and rotator cuff (RTC) weakness (compromised root branches) can lead to musculoskeletal shoulder disorders such as RTC tendonitis and adhesive capsulitis. Damage to the cervical plexus and its branches, as well as the cervical root branches, can cause neuropathic pain.

Apart from very early cases, radiotherapy is the most effective curative treatment decision. Though the results are poorer with bigger tumours, these are normally treatable with EBRT. Patients suffering from stage III or IV disease are normally managed with concomitant chemoradiation using cisplatin, which is validated in studies showing better results than sole radiation therapy. Radiation can damage any structure in the radiation field including nerve, muscle, tendon, ligament, blood vessel, lymphatic, and bone. Late effects of radiation in HNC include radiculopathy, cervical and/or brachial plexopathy, and mononeuropathies of the cranial and other nerves located within the radiation field. Late effects of radiation include dysphagia, dysarthria, xerostomia, trismus, dropped head syndrome, cervical dystonia, shoulder dysfunction, neck and/or shoulder pain, and multiple other adverse sequelae. The combination of surgery and radiation likely has additive morbidity. The treatment of late effects in HNC survivors varies depending on the specific issues. Physical therapy is the primary modality for most disorders. Nociceptive pain (RTC tendonitis, adhesive capsulitis) not responsive to physical therapy may require nonsteroidal antiinflammatory drugs (NSAIDs) or opioid analgesics.

In India, most patients are in advanced stage at presentation where concurrent chemo-radiation is the best available treatment. Yet, 5-year DFS is only about 59.1\% in spite of this treatment. [2] Due to large patient population and lesser availability of radiotherapy equipment, there is often deferral in start of radiotherapy for them. Hence, neo-adjuvant chemotherapy is used by some oncologists before starting EBRT. Some oncologists prefer hypo fractionated radiation therapy over conventional fractionation. Since there is no rational comparative study between neoadjuvant chemotherapy or hypo fractionation and concurrent chemoradiation, we compared results of treatment with chemo-radiotherapy with that of neoadjuvant chemotherapy followed by hypo-fractionated radiotherapy in locally advanced head and neck carcinoma.

\section{METHODS}

This is a prospective randomized study comparing two commonly practiced treatment methods. Biopsy proved squamous carcinoma of larynx and pharynx excluding nasopharynx, stage III and IV excluding IVC (i.e., nonmetastatic, node positive or locally advanced disease) with normal routine investigations and renal and liver function tests, having good performance status and registered in between February and September 2016 were counted in in our study. It was done in Bankura Sammilani Medical College and Hospital, Bankura. All patients detected with head and neck cancer were planned to be counted in. Hence, sample-size calculation by Cochran's formula was not required. 142 patients were counted in this trial.

IEC clearance and informed consent in patient's own language were obtained. Each patient was randomized by blind-envelope technique to get either concurrent chemoradiation with a total radiotherapy dose of 70 Gy (in conventional fractionation using weekly injection cisplatin 40 $\mathrm{mg} / \mathrm{m}^{2}$ as radio-sensitizer), or three cycles of neoadjuvant chemotherapy with carboplatin AUC 6 plus injection paclitaxel $175 \mathrm{mg} / \mathrm{m}^{2}$ and injection $5 \mathrm{FU} 1000 \mathrm{mg} / \mathrm{m}^{2}$ day $1-4$ after hypofractionated EBRT (40 Gy over 16 fractions through three and half weeks followed by 20 Gy in 8 fractions over one and half weeks boost, started within 3 weeks of chemotherapy). During EBRT, each patient was treated based on imaging data with clinical correlation. Clinical assessment, blood, otorhinolaryngeal examination with endoscopy and imaging were done periodically during follow up when required. DFS at the completion of three years and treatment toxicities by Common Terminology Criteria for Adverse Events (CTCAE) v5.0 were recorded for all cases.

\section{Statistics}

The arms were analyzed by one way ANOVA using IBM SPSS Statistics ${ }^{\circledR}$ software v20. Toxicities were compared by Williams test. Value of $\mathrm{P}<0.05$ was declared to be significant statistically.

\section{RESULTS}

Complete response (CR) was a little better following neoadjuvant chemotherapy followed by hypo fractionated radiotherapy arm. Although statistically insignificant, locoregional toxicity and DFS were little greater after hypo fractionated radiation therapy. Documented late toxicities were within nephrotoxicity (Grade I-II) and loco-regional complications. Both treatment modalities showed equivalent result of efficacy and complications after an observation period of three years. In this study, characteristics of patients were comparable in both arms (Table 1). Although statistically insignificant, complete response was a bit higher with neoadjuvant chemotherapy followed by hypo fractionated radiotherapy arm (Table 2). DFS (Table 2) and loco-regional complications (Table 3) were a little higher in hypo 
fractionated radiation therapy arm. This difference too was not statistically significant. Late complications documented, were restricted to grade I-II renal toxicity and loco-regional complications. Hence, both treatment modalities resulted in equal results of effectiveness and complications when followed over a three year period.

\begin{tabular}{|c|c|c|c|}
\hline & $\begin{array}{c}\text { Concurrent Chemo- } \\
\text { Radiation Utilising } \\
\text { Conventional } \\
\text { Fractionation }\end{array}$ & $\begin{array}{c}\text { Hypofractionated } \\
\text { Radiation Therapy } \\
\text { after Neo-Adjuvant } \\
\text { Chemotherapy }\end{array}$ & $\mathbf{p}$ \\
\hline Number of patients & 69 & 73 & 0.60 \\
\hline Mean age & 50.5 yrs. (SD 6.45) & 53.5 yrs. (SD 7.82) & 0.98 \\
\hline Stage & 28 & 24 & \multirow{2}{*}{0.72} \\
\hline III & 29 & 37 & \\
\hline IVA & 12 & 12 & \\
\hline IVB & Table 1. Patient Characteristics \\
\hline \multicolumn{3}{|c|}{} \\
\hline
\end{tabular}

\begin{tabular}{|l|c|c|c|}
\hline & $\begin{array}{c}\text { Concurrent Chemo- } \\
\text { Radiation Utilising } \\
\text { Conventional } \\
\text { Fractionation }\end{array}$ & $\begin{array}{c}\text { Hypofractionated } \\
\text { Radiation Therapy } \\
\text { after Neo-Adjuvant } \\
\text { Chemotherapy }\end{array}$ & p Value \\
\hline CR after EBRT & 50 & 51 & 0.31 \\
\hline PR after EBRT & 19 & 22 & 0.25 \\
\hline DFS at 2 years & 67 & 70 & 0.27 \\
\hline \multicolumn{3}{|c|}{ Table 2. Assessment of Response } \\
\hline [DFS = disease free survival, PR = partial response, CR = complete response] \\
\hline
\end{tabular}

\begin{tabular}{|c|c|c|c|}
\hline & $\begin{array}{c}\text { Concurrent Chemo- } \\
\text { Radiation Utilising } \\
\text { Conventional } \\
\text { Fractionation }\end{array}$ & $\begin{array}{c}\text { Hypofractionated } \\
\text { Radiation Therapy } \\
\text { after Neo-Adjuvant } \\
\text { Chemotherapy }\end{array}$ & $\mathbf{p}$ \\
\hline Grade III acute toxicity & 11 & 16 & 0.63 \\
\hline Grade III late toxicity & 2 & 4 & 0.75 \\
\hline $\begin{array}{c}\text { Chemotherapy induced } \\
\text { nephrotoxicity }\end{array}$ & 1 & 0 & 0.60 \\
\hline \multicolumn{4}{|c|}{ Table 3. Toxicity Assessment } \\
\hline
\end{tabular}

\section{DISCUSSION}

Cancer of head and neck epitomizes about $5 \%$ of the all cancer sites. Around the world, over 500,000 patients are identified each year ${ }^{[4]}$ with 100,800 people living in Europe, and almost 40,000 of European patients die from the disease. [5] More men than women develop head and neck cancer; the incidence is rising $[5,6]$ but varies greatly across Europe. These cancers include locations with very good outcome (lips, $94 \%$ five-year life span), moderate outcome (salivary glands, 61\%; larynx, $62 \%$ ), fair outcome (oral cavity, $45 \%$; tongue, 39\%; nasopharynx, $43 \%$ and oropharynx, $32 \%$ ), and poor outcome (hypo-pharynx, 25\%).[4] The overall age-adjusted mortality rates from these cancers in the European Union decreased in the period between 1992 and 2002: oral cavity and pharynx ($8 \%)$, larynx $(-27 \%)$, thyroid $(-23 \%)$ in men and thyroid $(-$ $28 \%$ ) in women.[6]

Only radiation therapy and surgery can provide cure for these malignancies. Chemotherapy alone, although not curative, augments the results of radiotherapy and is regularly used in combination therapy in stage III -IV cancers. A recent analysis of institutions with high accrual or low accrual of patients in cooperative group studies found that patients treated in high-volume RT centres had better survival compared with patients treated at low-volume institutions, after accounting for RT protocol deviations.[7] Patients with low nutrition often need a nasogastric Ryle's tube or a per- cutaneous gastrostomy (PEG) before surgery or radiotherapy, especially if concurrent systemic therapy is utilised. Altered fractionation increases acute complications, whereas, late complications are equivalent with conventional fractionation. ${ }^{[8]}$ Hyper fractionation resulted in improved overall survival, whereas the concomitant boost schedule did not. Of note, when concurrent chemotherapy is added, there does not appear to be a tumour control advantage for use of altered fractionated compared to standard fractionation RT. [9] However, there are no randomized trials comparing hyper fractionation and concomitant chemotherapy with once-daily fractionation and concomitant chemotherapy.

Induction or neoadjuvant chemotherapy prior to local treatment has academic benefits. Goldie and Coldman model.[10] Of spontaneous mutations describes that the drug resistant fraction of cells increases with increase of tumour size. Initial chemotherapy lessens resistance. Other probable benefits of neoadjuvant chemo-therapy may be shrinking of the tumour and regional lymph nodes, thus helping subsequent surgery or radiotherapy attain local control and enabling surgery; identifying patients with response, whose disease can be controlled effectively by radiotherapy or surgery; selection of patient for help from added treatment following locoregional therapy; and management of occult metastasis. Probable risks are the deferral in locoregional management, which may cause tumour re-growth and spread; the selection of chemotherapy and radio-resistant tumour cells; and the increase of local complications through radiotherapy or surgery afterwards.

Many randomized studies examined induction chemotherapy in head and neck cancer patients. Only one study reported increase in survival in a subset of subjects with unresectable disease, while all others did not show an improvement in survival. Despite the high overall response rates, induction chemotherapy did not have an impact on locoregional control. However, there were less distant metastases as site of first failure. A meta-analysis of these studies could not show a survival benefit for cisplatin-based induction chemotherapy.[11] However, several studies evaluated induction chemotherapy with taxanes followed by chemo-radiation in patients with unresectable disease.[12-14] In the taxane arm the response rate, overall survival and DFS were higher than the non-taxane arm with acceptable toxicity. Based on these studies, chemo-radiation after taxane-based induction chemotherapy is the standard of care for unresectable disease.

But no study has compared results of hypo fractionation after neo-adjuvant chemotherapy to usual chemo-radiation. Our study compares this. It reveals similar patient characteristics in both arms (Table 1). Concurrent chemoradiation resulted in slightly better complete response. But this was statistically insignificant (Table 2). Locoregional complications and DFS (Table 2) were slightly better after hypofractionated radiotherapy (Table 3). This was also statistically not significant. Late toxicities were restricted to locoregional complications and nephrotoxicity (Grade I-II). Thus, both treatment modalities had similar result in regarding effectiveness and complications during follow up for three year. 


\section{CONCLUSIONS}

Hypo fractionated radiotherapy after neo-adjuvant chemotherapy is an appropriate substitute and is statistically non-inferior to the recommended practice of concurrent chemoradiation, in treatment centres overloaded with cancer patients. Drop-outs are lower and it appears to be convenient too.

\section{REFERENCES}

[1] American Cancer Society. Cancer Facts and Figures 2017. Atlanta, GA: American Cancer Society, 2017.

[2] Trotti A, Fu KK, Pajak TF, et al. Long term outcomes of RTOG 90-03: a comparison of hyperfractionation and two variants of accelerated fractionation to standard fractionation radiotherapy for head and neck squamous cell carcinoma. Int J Radiat Oncol Biol Phys 2005;63(Suppl 1):S70-S1.

[3] Siegel RL, Miller KD, Jemal A. Cancer statistics, 2016. CA Cancer J Clin 2016;66(1):7-30.

[4] Vokes EE, Cohen EE, Grandis JR. Introduction: head and neck cancer. Semin Oncol 2008;35(3):196-7.

[5] Sant M, Aareleid T, Berrino F, et al. EUROCARE-3: survival of cancer patients diagnosed 1990-94--results and commentary. Ann Oncol 2003;14(Suppl 5):v61-118.

[6] Bosetti C, Bertuccio P, Levi F, et al. Cancer mortality in the European Union, 1970-2003, with a joinpoint analysis. Ann Oncol 2008;19(4):631-40.
[7] Wuthrick EJ, Zhang Q, Machtay $M$, et al. Institutional clinical trial accrual volume and survival of patients with head and neck cancer. J Clin Oncol 2015;33(2):156-4.

[8] Mendenhall WM, Riggs CE, Vaysberg M, et al. Altered fractionation and adjuvant chemotherapy for head and neck squamous cell carcinoma. Head Neck 2010;32(7):939-45.

[9] Bourhis J, Sire C, Graff P, et al. Concomitant chemoradiotherapy versus acceleration of radiotherapy with or without concomitant chemotherapy in locally advanced head and neck carcinoma (GORTEC 99-02): an open-label phase 3 randomised trial. Lancet Oncol 2012;13(2):145-53.

[10] Goldie JH, Coldman AJ. A mathematic model for relating the drug sensitivity of tumors to their spontaneous mutation rate. Cancer Treat Rep 1979;63(11-12):172733.

[11] Specenier PM, Vermorken JB. Neoadjuvant chemotherapy in head and neck cancer: should it be revisited? Cancer Lett 2007;256(2):166-77.

[12] Hitt R, Lopez-Pousa A, Martinez-Trufero J, et al. Phase III study comparing cisplatin plus fluorouracil to paclitaxel, cisplatin and fluorouracil induction chemotherapy followed by chemoradiotherapy in locally advanced head and neck cancer. J Clin Oncol 2005;23(34):8636-45.

[13] Vermorken JB, Remenar E, Van Herpen C, et al. Cisplatin, fluorouracil and docetaxel in unresectable head and neck cancer. N Engl J Med 2007;357(17):1695-704.

[14] Posner MR, Hershock DM, Blajman CR et al. Cisplatin and fluorouracil alone or with docetaxel in head and neck cancer. N Engl J Med 2007;357(17):1705-15. 